\title{
Introduction to the Special Issue on Machine Learning for CAD
}

The idea of this special issue had stemmed from a workshop that we organized at the Design, Automation, and Test in Europe (DATE) conference in March 2019. The workshop back then aimed at putting the initial seeds for a new research community that collects experts in CAD with a special focus on machine learning (ML) from both industrial as well as academic fields. The workshop later turned into a regular workshop sponsored by IEEE and ACM called MLCAD: http://mlcad.itec.kit.edu, and the first edition was held in September 2019 in Canada.

Advances in ML over the past half-dozen years promise to revolutionize the effectiveness of ML in a large variety of domains. However, design processes present challenges that require parallel advances in ML and CAD as compared to traditional ML applications such as image classification. $\mathrm{CAD}$ in this context is broadly defined as design-time techniques as well as run-time techniques. In this context, this special issue on ML for CAD focuses on introducing, exploring, and investigating the current as well as future challenges and opportunities when ML and CAD come together.

One of the key goals of this special issue is to offer the readers, who are not specialists in ML or may not even have a specific background, a new perspective of the varied ongoing efforts in research that aim at employing ML techniques and algorithms, evolved over decades, in supporting $\mathrm{CAD}$ tools. Another goal is to demonstrate to readers how bringing ML and CAD together can open new doors in research toward increasing the efficiency of computing through advanced ML. This holds for both chip design as well as run-time management techniques. In particular, the special issue covers various abstraction layers. It demonstrates how ML does enrich both design-time as well as run-time CAD methodologies to significantly improve their effectiveness.

In this special issue, we have 12 interesting articles coving a wide range of different CAD areas. Starting from intelligent methods for chip testing and faults diagnosis, the articles "Toward Smarter Diagnosis: A Learning-Based Diagnostic Outcome Previewer" by Q. Huang et al., "FineGrained Adaptive Testing Based on Quality Prediction" by M. Liu, and "Machine Learning-Based Defect Coverage Boosting of Analog Circuits under Measurement Variations" by N. Xama et al. demonstrate how ML techniques can very effectively increase the yield of chips and help chips' designers to rapidly identify existing defects in both digital as well as analog circuits.

When it comes to FPGA chips, a new method to improve the routability using ML was proposed in "Improving FPGA-Based Logic Emulation Systems through Machine Learning" by H. Szentimrey et al. In addition, A. Agnesina et al. demonstrated the role that ML may play in emulations in their article "Improving FPGA-Based Logic Emulation Systems through Machine Learning."

One of the major challenges that faces designers in the nano-CMOS era is improving the reliability and security of on-chip systems in which the effects of circuit's aging as well as the threats of adversarial attacks are kept at bay. To this end, "Machine Learning Approach for Fast

\footnotetext{
ACM Reference format: https://doi.org/10.1145/3410864

(c) 2020 Copyright held by the owner/author(s).

1084-4309/2020/10-ART36

https://doi.org/10.1145/3410864
}

Jörg Henkel, Hussam Amrouch, and Marilyn Wolf. 2020. Introduction to the Special Issue on Machine Learning for CAD. ACM Trans. Des. Autom. Electron. Syst. 25, 5, Article 36 (October 2020), 2 pages. 
Electromigration-Aware Aging Prediction in Incremental Design of Large Scale On-Chip Power Grid Network" by S. Dey et al. employed ML to accurately estimate the effects of electromigration aging in metal interconnects across the chip. When it comes to security, "Adversarial Perturbation Attacks on ML-Based EDA: A Case Study on CNN-Based Lithographic Hotspot Detection" by K. Liu et al. proposed an adversarial retraining strategy to improve the robustness of CNN-based hotspot detection and showed how such a strategy significantly improves robustness against adversarial attacks without compromising the accuracy of classification.

Performing accurate simulations is one of the primary objectives of any CAD tool. "Predicting Memory Compiler Performance Outputs Using Feed-Forward Neural Networks” by F. Last et al. showed how ML can help in estimating the performance of memory compilers and "NeuPow: A CAD Methodology for High-Level Power Estimation Based on Machine Learning" by Y. Nasser et al. explored the benefits of ML in increasing the accuracy of high-level power estimations. In addition, "Multi-Fidelity Surrogate-Based Optimization for Electromagnetic Simulation Acceleration" by Y. Wang et al. studied how ML helps in accelerating electromagnetic simulations, which are essential for analog RF circuit design to ensure proper functionality during operation.

Finally, early exploration during the design time is always vital to improve the optimality. "PREASC: Automatic Portion Resilience Evaluation for Approximating SystemC-based Designs Using Regression Analysis Techniques” by M. Goli et al. proposed a new ML-based framework to identify the parts of design that are more resilient to errors in order to apply approximation. Finally, "Machine Learning Approaches for Efficient Design Space Exploration of ApplicationSpecific NoCs" by Y. Hu et al. proposed a new approach that employs ML in order to perform rapid design-space exploration for Network-on-Chip in MPSoCs.

We would like to thank all authors for their valuable contributions as well as all reviewers for their help and support. This special issue would not be possible without the outstanding work from both authors and reviewers.

\author{
Jörg Henkel \\ Hussam Amrouch \\ Marilyn Wolf \\ Guest Editors
}

